\title{
Adjuvant hypofractionated partial-brain radiation therapy for pediatric Ewing sarcoma brain metastases: case report
}

\author{
Ritchell van Dams, BA, ${ }^{1}$ Henry S. Park, MD, MPH, ${ }^{1}$ Ahmed K. Alomari, MD, ${ }^{2}$ \\ Adele S. Ricciardi, BS, ${ }^{3}$ Harini Rao, MD, ${ }^{4}$ Joseph McNamara, MD, ${ }^{4}$ Michael L. DiLuna, MD, ${ }^{3,4}$ and \\ Ranjit S. Bindra, MD, PhD',2 \\ Departments of ${ }^{1}$ Therapeutic Radiology, ${ }^{2}$ Pathology, ${ }^{3}$ Neurosurgery, Section of Pediatric Neurosurgery, and ${ }^{4}$ Pediatrics, Section \\ of Pediatric Hematology and Oncology, Yale School of Medicine, New Haven, Connecticut
}

\begin{abstract}
This case report demonstrates that hypofractionated partial-brain radiation therapy with limited margins is a reasonable approach following gross tumor resection of Ewing sarcoma metastases to the brain. The patient presented with 2 intracranial metastases treated with gross-total resection followed by radiation therapy to $30 \mathrm{~Gy}$ in 5 fractions. The patient experienced symptomatic treatment-related inflammatory changes with resolution after receiving dexamethasone. He remains alive at 21 months of follow-up with no evidence of disease.
\end{abstract}

http://thejns.org/doi/abs/10.3171/2015.8.PEDS15313

KEY WORDS Ewing sarcoma; brain metastases; radiation therapy; radiation necrosis; oncology

$\mathrm{E}$ WING sarcoma is infrequently associated with CNS metastases, and the optimal management of these intracranial lesions is unclear. The reported median survival after diagnosis of brain metastases is between 2.7 and 12 months. Here, we present the case of a pediatric patient with 2 brain metastases from Ewing sarcoma that were treated with resection and adjuvant hypofractionated partial-brain radiation therapy to $30 \mathrm{~Gy}$ in 5 fractions.

\section{Case Report}

This 9-year-old boy had a history of Ewing sarcoma of the left distal femur that was diagnosed in July 2011. He underwent neoadjuvant chemotherapy, limb-sparing resection in December 2011, and adjuvant chemotherapy (total 17 cycles of vincristine, doxorubicin, cyclophosphamide, irinotecan, and etoposide) completed in April 2012. No diagnostic lumbar punctures were performed after definitive therapy, and the patient never received intrathecal chemotherapeutic agents. In March 2013, he presented with a 2-week history of recurrent headaches, after which he developed nausea, vomiting, right-hand numbness and weakness, and gait instability. Brain MRI performed in a different country revealed a dominant $4 \times 4-\mathrm{cm}$ left frontoparietal mass associated with a $0.5-\mathrm{cm}$ midline shift, as well as an adjacent $2 \times 2-\mathrm{cm}$ left frontal mass (Fig. 1A and $\mathrm{B}$ ).

The following day, the patient underwent resection of the 2 intracranial masses. The tumors were found to be soft and highly vascularized, with bloody cystic areas deep within the dominant tumor cavity. Gross-total resection (GTR) was performed without significant complications, and no residual disease was found on postoperative MRI (Fig. 1C and D). Histopathological examination showed a highly cellular tumor with necrosis, numerous mitoses, and CD99 immunostain positivity. These findings were consistent with the metastasis from the patient's known Ewing sarcoma (Fig. 2). Whole-body PET-CT scanning was performed 4 days after resection. There was no evidence of widespread dissemination at that time.

The patient was subsequently treated with partial-brain external-beam radiation therapy to a dose of 30 Gy in 5 fractions to the resection cavity (Fig. 3). Chemotherapy was started the week after radiation therapy concluded and consisted of vincristine, temozolomide, and irinote-

ABBREVIATIONS GTR = gross-total resection; WBRT = whole-brain radiation therapy.

SUBMITTED May 19, 2015. ACCEPTED August 10, 2015.

INCLUDE WHEN CITING Published online December 4, 2015; DOI: 10.3171/2015.8.PEDS15313. 

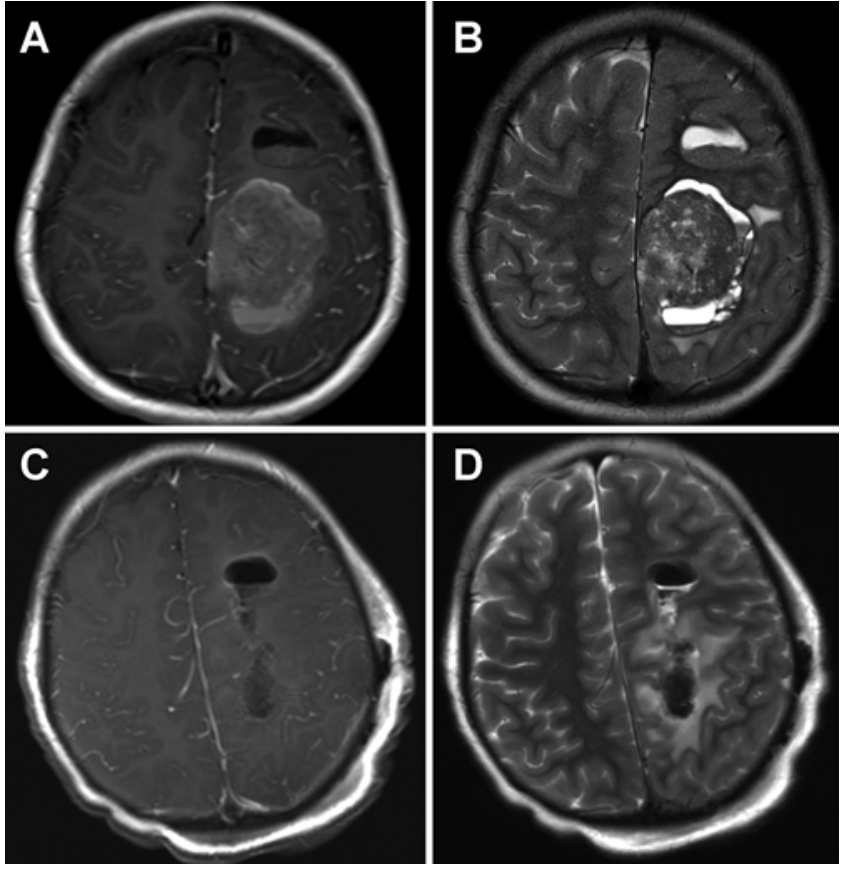

FIG. 1. Axial postcontrast T1-weighted (A) and T2-weighted (B) MR images demonstrating the metastatic Ewing sarcoma tumors within the left frontal and parietal lobes with heterogeneous enhancement $(A)$ and cystic components (B). Postoperative axial postcontrast T1-weighted (C) and T2-weighted (D) MR images showing GTR.

can for 12 cycles. MRI performed in July 2013 showed 2 small areas of enhancement in the left paracentral lobule in the surgical cavity as well anteriorly in the left frontal lobe both within the treatment field, which remained stable in August 2013 and enlarged slightly in November 2013 (not shown). It was unclear at the time whether this represented recurrence or treatment-related inflammatory changes. In December 2013, the patient presented with sudden-onset numbness and weakness in the right fourth and fifth digits and was found to have a new, right pronator drift. Brain MRI showed that the enhancing area in the left frontal lobe had increased to $3 \mathrm{~cm}$ with extensive perilesional edema causing midline shift and subfalcine herniation (Fig. 4A and B). MR multivoxel spectroscopy was performed the following day and showed no definitive intralesional increased vascularity (Fig. 4C), prominent lactate peaks in lesional and perilesional regions, and elevated choline levels at the posterior medial aspect of the enhancing lesion. This pattern was considered to be consistent with a diagnosis of treatment-related inflammatory changes rather than recurrence of disease. The patient's symptoms resolved after he received dexamethasone, and he completed his chemotherapy regimen in January 2014. MRI in late January 2014 demonstrated significant improvement in the size of the enhancing lesion, perilesional edema, and mass effect. In April 2014, the patient experienced a generalized tonic-clonic seizure followed by frequent focal partial seizures and recurrence of his right-sided numbness and weakness. MRI was performed and showed an increase in the size of the lesion with significant surrounding FLAIR signal (Fig. 4D and E). MR perfusion now showed increased perfusion in the posterior aspect of the lesion, raising suspicion for recurrence of the metastatic disease (Fig. 4F). A subsequent stereotactic biopsy performed via open craniotomy confirmed radiation necrosis with no evidence of disease (Fig. 5). Although no formal cognitive testing was performed, care workers and clinicians (including a pediatric neurologist) consistently noted the achievement of developmental milestones and the absence of cognitive and motor delays. As of last follow-up in December 2014, the patient was found to have no clinical or radiographic evidence of disease, resolving radiation necrosis, and no apparent neurocognitive longterm side effects from radiation therapy.

\section{Discussion}

Ewing sarcoma is a rare bone tumor of neuroectodermal origin. Marsa and Johnson first described isolated metastases to the CNS in 1971, and their hypothesis was that systemic chemotherapeutic suppression allowed the CNS micrometastases time to grow and present clinically. ${ }^{10}$ Mehta and Hendrickson reported that 15 of 27 (56\%) patients with Ewing sarcoma had neurological signs and symptoms presumed to be caused by CNS metastases. ${ }^{11}$ The conclusion drawn at the time was that patients with Ewing sarcoma would benefit from prophylactic radiation and chemotherapy to these "sanctuary" sites.
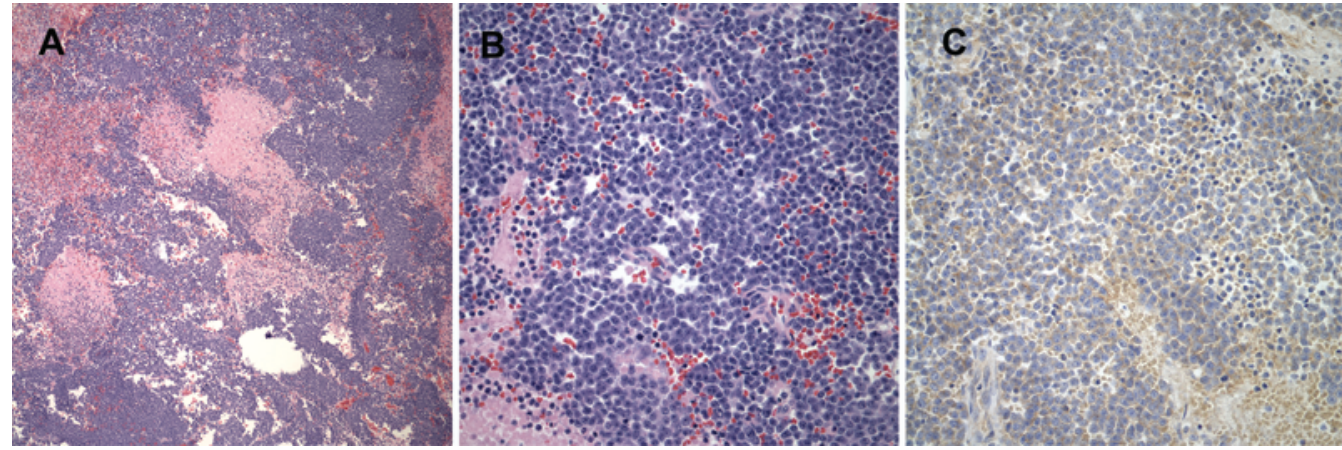

FIG. 2. A: Representative H \& E-stained section from the initial resection depicting diffuse growth of neoplastic cells with geographic areas of necrosis. B: On higher magnification, the tumoral cells show cellular crowding, high nuclear/cytoplasmic ratio, nuclear hyperchromasia, and brisk mitoses. C: Immunohistochemical staining for CD99 demonstrates moderate-intensity cytoplasmic and membranous patterns. Original magnification $\times 10(\mathrm{~A}) ; \times 40(\mathrm{~B}$ and $\mathrm{C})$. Figure is available in color online only. 

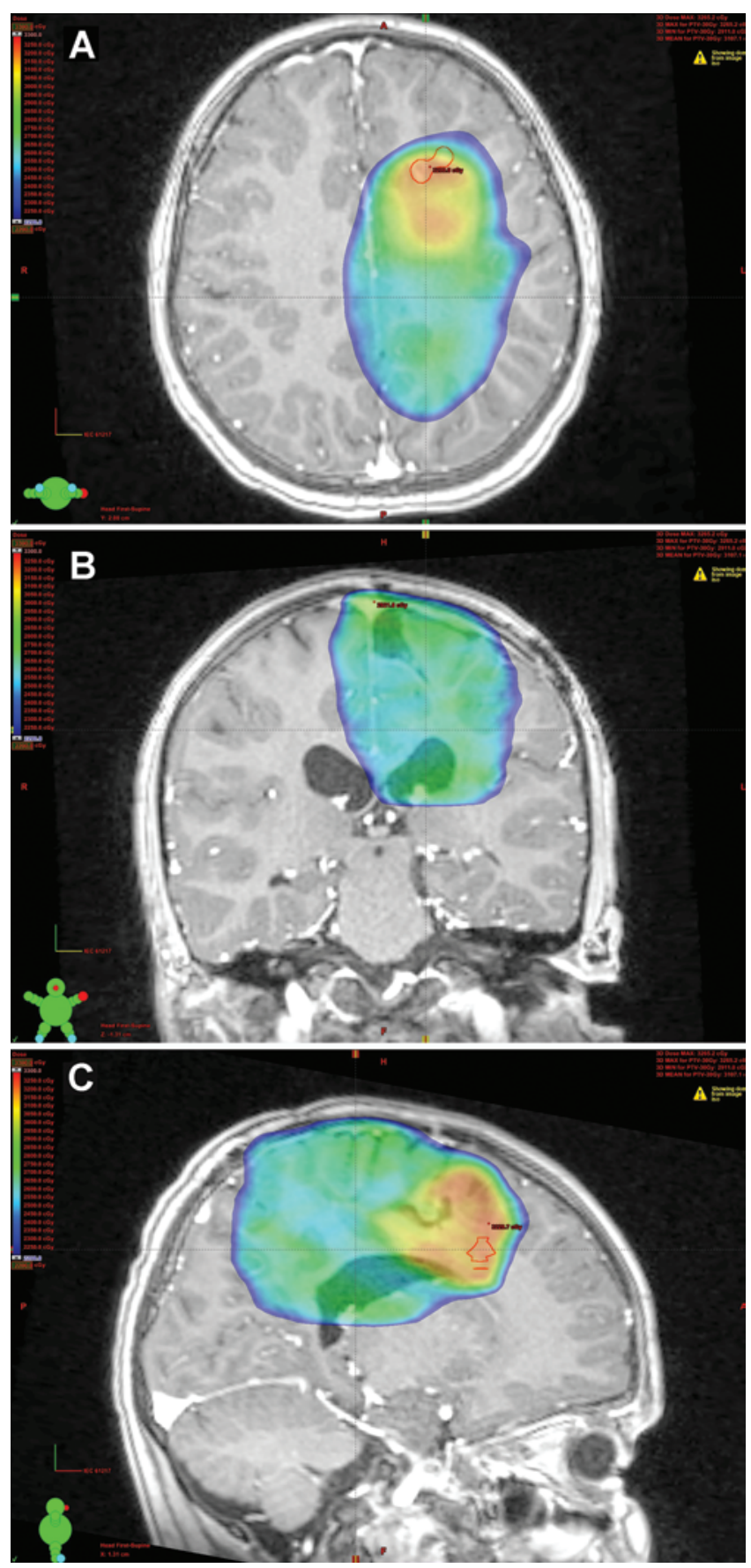

FIG. 3. Axial (A), coronal (B), and sagittal (C) views of the isodose distribution of the patient's 3D conformal treatment to the metastasis region and margin to $3000 \mathrm{cGy}$ in 5 fractions. Figure is available in color online only.

This paradigm was challenged by larger reviews of patient registries with a focus on imaging- or autopsy-proven metastasis. A review of 134 patients with Ewing sarcoma showed evidence of CNS metastasis in 3 patients (2.2\%), and in all cases there was evidence of widespread metastasis before the CNS was involved. 7 A later retrospective review of 445 patients treated at the National Cancer In- stitute and the Intergroup Ewing's Sarcoma Study showed that true CNS metastasis occurred in 10 patients $(2.2 \%)$ and that the CNS was the site of first relapse in $3(0.7 \%){ }^{17}$ Trigg et al. showed that the lifetime risk of CNS involvement in a patient with Ewing sarcoma was approximately $10 \%$, and that the incidence was not affected by prophylactic radiation therapy or intrathecal methotrexate..$^{18}$

Given this, our patient was neither treated prophylactically nor screened regularly for CNS metastasis in the absence of any other signs of disease in the body. Prior case reports of radiotherapy for CNS metastases from Ewing sarcoma are summarized in Table 1 . Three notable cases reported long-term survival after GTR with adjuvant chemoradiation therapy, with no evidence of disease at 19 months or at 3.4 and 11 years of follow-up. ${ }^{8,12,13}$ Radiation therapy has traditionally been external-beam wholebrain radiation therapy (WBRT) of a dose between 30 and $60 \mathrm{~Gy}$, and occasionally with a local boost. ${ }^{13,15}$ However, Krasin et al. showed that $3 \mathrm{D}$ conformal radiation therapy and intensity-modulated radiation therapy with limited margins (clinical tumor volume based on a $2.0-\mathrm{cm}$ margin around the gross tumor volume) were adequate treatment options for nonrhabdomyosarcoma soft-tissue sarcomas. ${ }^{9}$ On this basis, a decision was made to limit treatment of the patient's isolated metastases to a 3D conformal plan with limited margins.

The presence of symptomatic radiation necrosis in our patient after hypofractionated partial-brain radiotherapy warrants a review of the literature to identify potential future avoidance strategies. Aoki et al. provided one of the earliest series regarding hypofractionated radiotherapy for previously unresected or radiation-treated brain metastases. $^{3}$ Among 45 patients treated with 18-35 Gy in 3-5 fractions, there were no cases of radiation necrosis in this series. Two subsequent studies further refined the treatment approach. The first identified 42 patients (22 of whom had metastases resected and 22 of whom underwent prior radiotherapy) compared several hypofractionated radiotherapy schedules. ${ }^{5}$ The symptomatic radiation necrosis rate was noted for 3 patients (7\%), but whether these patients had received prior radiotherapy was not specified. The authors ultimately recommended $30 \mathrm{~Gy}$ in 5 fractions as the future standard for hypofractionated radiotherapy for brain metastases. Another series published that year identified 30 patients who received hypofractionated radiotherapy, all of whom had undergone prior resection. ${ }^{2}$ In this report, there were no patients with radiation necrosis in the group who had never received WBRT (20 patients), while 3 patients (30\%) of the prior-WBRT group had treatment-related toxicity. Lastly, a recent study of 65 patients whose postsurgical cavities were treated with 25-30 Gy in 5 fractions found a radiation necrosis rate of 1.5\%. ${ }^{1}$ Taken as a whole, the presented literature suggests that the use of 30 Gy in 5 fractions to treat resection cavities for brain metastases is effective and offers minimal risk of toxicity, especially in a population that has never received intracranial radiotherapy. Despite this, the impact of this dose-fractionation schedule on toxicities like radiation necrosis in children with brain metastases warrants further investigation.

In summary, we describe a 9-year-old boy with 2 brain 

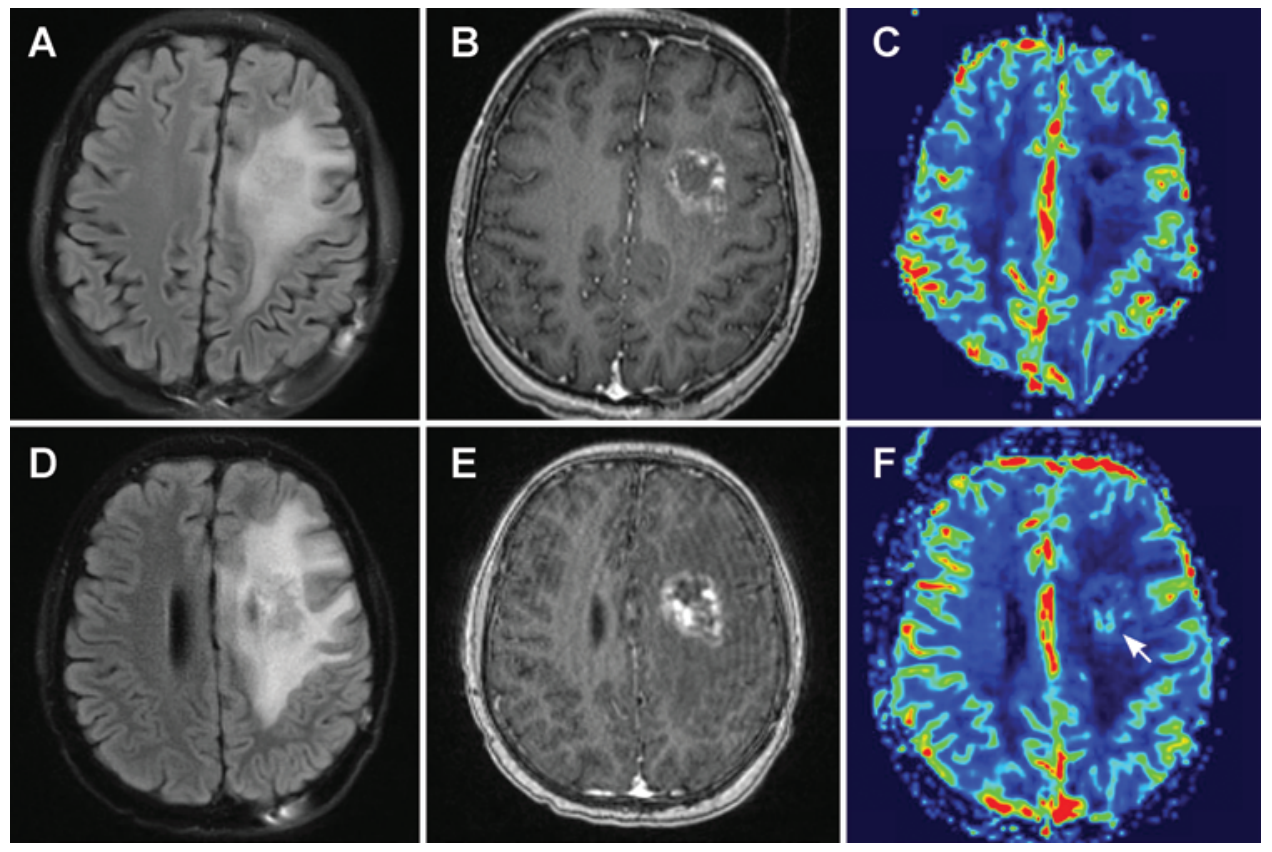

FIG. 4. Axial FLAIR (A), T1-weighted with contrast (B), and perfusion (C) MR images demonstrating new enhancement with increased FLAIR signal 8 months after treatment within the left frontal and parietal lobes without evidence for increased perfusion (C). Fourteen months after treatment, follow-up axial FLAIR (D), T1-weighted with contrast (E), and perfusion (F) MR images show marked increase in FLAIR signal, enhancement, and now increased perfusion (arrow), which is concerning for recurrence or treatment-related inflammatory changes. Figure is available in color online only.

metastases from Ewing sarcoma treated with resection and adjuvant hypofractionated partial-brain radiation therapy to $30 \mathrm{~Gy}$ in 5 fractions, rather than WBRT. Eight months after radiation therapy, the patient experienced symptomatic treatment-related inflammatory changes within the high-dose region of the treatment field that was confirmed on MR spectroscopy, with rapid symptom resolution after receiving dexamethasone. There is no consensus in the literature, but recent reports suggest that MR spectroscopy and perfusion-weighted imaging have higher sensitivity and specificity than PET-CT in differentiating radiation necrosis from recurrent metastasis. ${ }^{6}$ Biopsy remains the gold standard. Five months after this episode, new neurological symptoms prompted MR perfusion-weighted imaging, and the findings were suggestive of recurrence. A subsequent biopsy confirmed radiation necrosis with no evidence of disease. The patient was found to have resolving posttreatment changes with no evidence of disease as of his last follow-up 21 months after the presentation of his brain metastases. We demonstrate that hypofractionated partial-brain radiation therapy with limited margins is a reasonable approach following gross tumor resection, especially for pediatric patients who could potentially be spared the long-term neurocognitive side effects of WBRT.
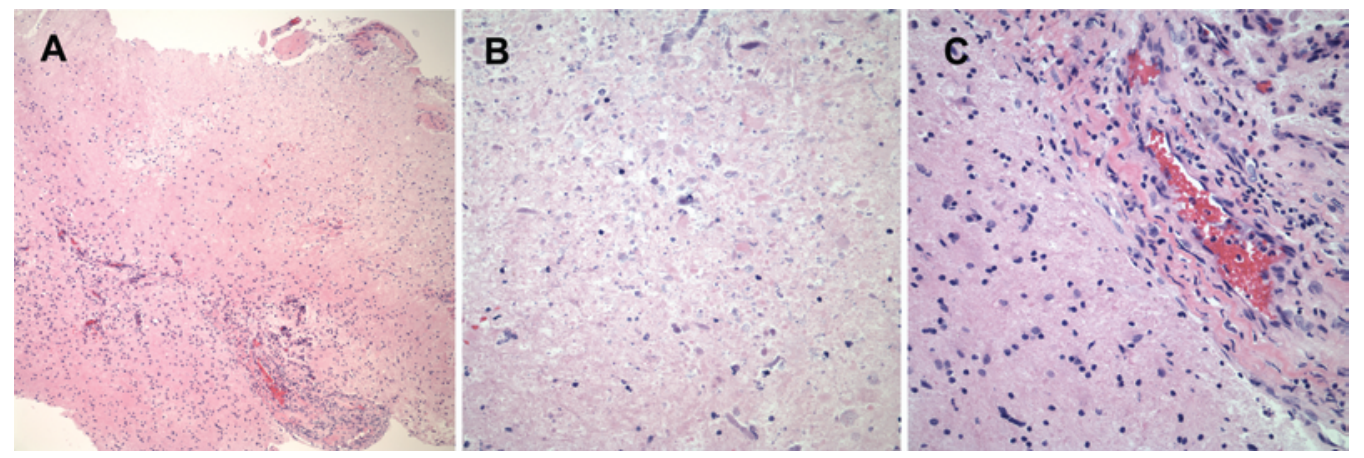

FIG. 5. A: Representative H \& E-stained section of a biopsy specimen of the area of increased perfusion postirradiation demonstrating areas of coagulative necrosis (upper right) and areas of demyelination and inflammatory vasculitis (lower left). B: On higher magnification, the necrotic areas show cellular debris and no residual viable tumoral cells. C: The area surrounding the necrotic focus shows demyelination (lower left) and marked lymphocytic vasculitis consistent with irradiation-induced changes. Original magnification $\times 10(\mathrm{~A}) ; \times 40(\mathrm{~B}$ and $\mathrm{C})$. Figure is available in color online only. 
TABLE 1. Comparison of case reports of radiotherapy for CNS metastases from Ewing sarcoma

\begin{tabular}{|c|c|c|c|c|c|c|}
\hline Authors \& Year & $\begin{array}{c}\text { No. of } \\
\text { Patients }\end{array}$ & $\begin{array}{c}\text { Yrs } \\
\text { Treated }\end{array}$ & $\begin{array}{c}\text { Treatment } \\
\text { (no. of patients) }\end{array}$ & Dose & Volume & Survival \\
\hline $\begin{array}{l}\text { Marsa \& Johnson, } \\
\quad 1971\end{array}$ & 1 & 1969 & $\mathrm{RT}$, chemo & 30 Gy & WBRT & $\begin{array}{l}2 \text { mos, died of systemic disease \& persistent } \\
\text { brain metastasis }\end{array}$ \\
\hline Sonntag \& Stein, 1978 & 1 & 1976 & GTR, RT & $40 \mathrm{~Gy}$ & WBRT & None reported \\
\hline Trigg et al., 1982 & 1 & 1979 & RT, chemo & $30 \mathrm{~Gy}$ & WBRT & 14 mos \\
\hline Simpson et al., 1989 & 1 & NR & RT & 30 Gy + 14.4 Gy & WBRT/boost & $\geq 2 \mathrm{mos}$ \\
\hline Colak et al., 1991 & 1 & 1973-1989 & RT & $50 \mathrm{~Gy}$ & WBRT & $\geq 4 \mathrm{mos}$ \\
\hline Olivi et al., 1991 & 1 & 1989 & GTR, RT, chemo & $55 \mathrm{~Gy}$ & WBRT & NED $\geq 19$ mos \\
\hline \multirow{3}{*}{$\begin{array}{l}\text { Parasuraman et al., } \\
1999\end{array}$} & \multirow[t]{3}{*}{9} & \multirow[t]{3}{*}{ 1962-1998 } & $\mathrm{RT}(4)$ & $20-46.8$ Gy & WBRT & $1-112$ days \\
\hline & & & RT, chemo (4) & 20-46.8 Gy & WBRT & $2-21 \mathrm{mos}$ \\
\hline & & & GTR, RT, chemo (1) & 30 Gy + 5 Gy & WBRT/boost & NED $\geq 112$ mos \\
\hline Koontz et al., 2006 & 5 & 1980-2002 & GTR, RT, chemo & NR & WBRT & 1 patient alive at $3.4 \mathrm{yrs}$ \\
\hline Paulino et al., 2013 & 2 & $1976-2001$ & $\mathrm{RT}$, chemo & $40-60 \mathrm{~Gy}$ & WBRT & $1.5-3$ mos, died of pulmonary metastases \\
\hline
\end{tabular}

Chemo = chemotherapy; NED = no evidence of disease; NR = not reported; RT = radiation therapy.

\section{References}

1. Ahmed KA, Freilich JM, Abuodeh Y, Figura N, Patel N, Sarangkasiri S, et al: Fractionated stereotactic radiotherapy to the post-operative cavity for radioresistant and radiosensitive brain metastases. J Neurooncol 118:179-186, 2014

2. Al-Omair A, Soliman H, Xu W, Karotki A, Mainprize T, Phan N, et al: Hypofractionated stereotactic radiotherapy in five daily fractions for post-operative surgical cavities in brain metastases patients with and without prior whole brain radiation. Technol Cancer Res Treat 12:493-499, 2013

3. Aoki M, Abe Y, Hatayama Y, Kondo H, Basaki K: Clinical outcome of hypofractionated conventional conformation radiotherapy for patients with single and no more than three metastatic brain tumors, with noninvasive fixation of the skull without whole brain irradiation. Int J Radiat Oncol Biol Phys 64:414-418, 2006

4. Colak A, Berker M, Ozcan OE, Erbengi A: CNS involvement in Ewing's sarcoma. A report of 12 cases. Acta Neurochir (Wien) 113:48-51, 1991

5. Eaton BR, Gebhardt B, Prabhu R, Shu HK, Curran WJ Jr, Crocker I: Hypofractionated radiosurgery for intact or resected brain metastases: defining the optimal dose and fractionation. Radiat Oncol 8:135, 2013

6. Kickingereder P, Dorn F, Blau T, Schmidt M, Kocher M, Galldiks N, et al: Differentiation of local tumor recurrence from radiation-induced changes after stereotactic radiosurgery for treatment of brain metastasis: case report and review of the literature. Radiat Oncol 8:52, 2013

7. Kies MS, Kennedy PS: Central nervous system involvement in Ewing's sarcoma. Ann Intern Med 89:226-227, 1978

8. Koontz BF, Clough RW, Halperin EC: Palliative radiation therapy for metastatic Ewing sarcoma. Cancer 106:17901793, 2006

9. Krasin MJ, Davidoff AM, Xiong X, Wu S, Hua CH, Navid $\mathrm{F}$, et al: Preliminary results from a prospective study using limited margin radiotherapy in pediatric and young adult patients with high-grade nonrhabdomyosarcoma soft-tissue sarcoma. Int J Radiat Oncol Biol Phys 76:874-878, 2010

10. Marsa GW, Johnson RE: Altered pattern of metastasis following treatment of Ewing's sarcoma with radiotherapy and adjuvant chemotherapy. Cancer 27:1051-1054, 1971

11. Mehta Y, Hendrickson FR: CNS involvement in Ewing's sarcoma. Cancer 33:859-862, 1974
12. Olivi A, Donehower RC, Mann RB, Brem H: Solitary, isolated metastasis from Ewing's sarcoma to the brain: case report. Surg Neurol 35:239-243, 1991

13. Parasuraman S, Langston J, Rao BN, Poquette CA, Jenkins JJ, Merchant T, et al: Brain metastases in pediatric Ewing sarcoma and rhabdomyosarcoma: the St. Jude Children's Research Hospital experience. J Pediatr Hematol Oncol 21:370-377, 1999

14. Paulino AC, Mai WY, Teh BS: Radiotherapy in metastatic Ewing sarcoma. Am J Clin Oncol 36:283-286, 2013

15. Simpson RK Jr, Bruner JM, Leavens ME: Metastatic Ewing's sarcoma to the brain: case report and review of treatment. Surg Neurol 31:234-238, 1989

16. Sonntag VKH, Stein BM: Intracerebral Ewing's sarcoma: case report. Neurosurgery 2:55-57, 1978

17. Trigg ME, Glaubiger D, Nesbit ME Jr: The frequency of isolated CNS involvement in Ewing's sarcoma. Cancer 49:2404-2409, 1982

18. Trigg ME, Makuch R, Glaubiger D: Actuarial risk of isolated CNS involvement in Ewing's sarcoma following prophylactic cranial irradiation and intrathecal methotrexate. Int J Radiat Oncol Biol Phys 11:699-702, 1985

\section{Disclosures}

The authors report no conflict of interest concerning the materials or methods used in this study or the findings specified in this paper.

\section{Author Contributions}

Conception and design: Bindra. Acquisition of data: van Dams, Park, Alomari, Ricciardi, DiLuna. Analysis and interpretation of data: van Dams. Drafting the article: van Dams, Park. Critically revising the article: all authors. Reviewed submitted version of manuscript: all authors. Approved the final version of the manuscript on behalf of all authors: Bindra. Study supervision: Bindra, Park, DiLuna.

\section{Correspondence}

Ranjit S. Bindra, Departments of Therapeutic Radiology and Pathology, Yale School of Medicine, 15 York St., 139 HRT, New Haven, CT 06511.email: ranjit.bindra@yale.edu. 